\title{
Effectiveness of COVID-19 Convalescent Plasma Infusion Within 48 Hours of Hospitalization With SARS-CoV-2 Infection
}

Natalia Lattanzio ${ }^{1}$, Cristina Acosta-Diaz ${ }^{1}$, Ricardo J. Villasmil ${ }^{1}$, Zachary Kirkland ${ }^{1}$, Caitlin Bass ${ }^{2}$, Sage Yenari $^{3}$, Jorge Conte ${ }^{1}$, Kevin Dawkins ${ }^{1}$, Tamela Fonseca ${ }^{4}$, Cindy Grimes ${ }^{2}$, Angie Stewart ${ }^{5}$, Mary E. Geary ${ }^{6}$, Harold Vore ${ }^{7}$, Karen Hamad ${ }^{1}$, Wilhelmine Wiese-Rometsch ${ }^{1}$, James Fiorica ${ }^{8}$, Manuel Gordillo ${ }^{9}$, Roberto Mercado ${ }^{9}$, Kirk Voelker ${ }^{10}$

1. Internal Medicine, Sarasota Memorial Hospital, Florida State University College of Medicine, Sarasota, USA 2. Internal Medicine, Sarasota Memorial Hospital, Sarasota, USA 3. Medicine, Sarasota Memorial Hospital, Sarasota, USA 4. Clinical Research, Sarasota Memorial Hospital, Sarasota, USA 5. Clinical Database Administration, Sarasota Memorial Hospital, Sarasota, USA 6. Quality Improvement, Sarasota Memorial Hospital, Sarasota, USA 7. Pathology, Sarasota Memorial Hospital, Sarasota, USA 8. Oncology, Sarasota Memorial Hospital, Sarasota, USA 9. Infectious Disease, Sarasota Memorial Hospital, Sarasota, USA 10. Pulmonology and Critical Care, Sarasota Memorial Hospital, Sarasota, USA

Corresponding author: Natalia Lattanzio, natalia-lattanzio@smh.com

Review began 07/11/2021 Review ended 07/18/2021 Published 07/30/2021

\section{(c) Copyright 2021}

Lattanzio et al. This is an open access article distributed under the terms of the Creative Commons Attribution License CC-BY 4.0., which permits unrestricted use, distribution, and reproduction in any medium, provided the original author and source are credited.

\section{Abstract}

On January 30, 2020, the World Health Organization (WHO) declared the severe acute respiratory syndrome coronavirus 2 (SARS-CoV-2) pandemic a worldwide emergency. Worldwide there have been 170 million cases of the resulting disease coronavirus 2019 (COVID-19), of those, 3.53 million have resulted in death. The Food and Drug Administration (FDA) with Mayo Clinic as the lead institution authorized COVID-19 convalescent plasma (CCP) for treatment of SARS-CoV-2 infection. Effective therapeutic window for CCP administration had yet to be defined. We addressed this gap by characterizing longitudinal biologic response and clinical outcomes of COVID-19 patients treated with CCP. Primary outcome was discharged to home/home health.

Categories: Internal Medicine, Infectious Disease, Public Health

Keywords: covid-19, convalescent plasma, sars-cov-2, discharge to home, infusion

\section{Introduction}

On January 30, 2020, the World Health Organization (WHO) declared the severe acute respiratory syndrome coronavirus 2 (SARS-CoV-2) pandemic a worldwide emergency. Worldwide there have been 114 million cases of the resulting disease coronavirus 2019 (COVID-19) of those, 2.54 million have resulted in death. At pandemic debut, available treatment was limited to supportive care as no approved therapy or vaccination was available. This treatment vacuum motivated the utilization of convalescent plasma infusion to complement the antibody response.

Passive immunization has been successfully used to treat infectious diseases since the 1890s [1,2]. Convalescent plasma (CP) was used to treat Spanish Influenza A (H1N1), severe acute respiratory syndrome in 2003 caused by SARS-associated coronavirus (SARS-CoV), and Influenza A pandemic in 2009 [3,4]. Underpinning CP treatment is that subjects recovering from viral infection marshalled an effective antibody response. And donated $\mathrm{CP}$ administered to an infected individual anticipating transfused antibodies would affect sufficient passive immunity to reduce symptoms and mortality risk.

Results from small case series conducted during the prior Middle East Respiratory Syndrome (MERS) and SARS-CoV documented CP to be safe, well-tolerated and promoted faster viral clearance, particularly when given early in the disease course [5]. The primary hypothesized mechanism of action of CP in COVID-19 pathogenesis involves antibody neutralization downregulating the hyperinflammatory response evoked by SARS-CoV-2 ribonucleic acid (RNA) [6,7]. Also, the thought was that transfused antibodies passively increased tissue repair decreasing and/or obviating complications and progression to death [8-11]. Additional preliminary clinical evidence suggested that CP might benefit individuals with SARS-CoV-2 infection and symptom onset suggestive of COVID-19.

The Food and Drug Association (FDA) with Mayo Clinic as the lead institution authorized COVID-19 convalescent plasma (CCP) for treatment of CoV-2 infection. Effective therapeutic window for CCP administration had yet to be defined. We addressed this gap by characterizing longitudinal biologic response and clinical outcomes of COVID-19 patients treated with CCP.

\section{Materials And Methods}




\section{Study design and population}

This retrospective longitudinal study analyzed electronic medical record data, including but not limited to characteristics and laboratory test findings from 197 patients consecutively admitted between March 28 and August 5, 2020. Among those, 92 and 105, respectively, received CCP infusion within $48 \mathrm{~h}$ of versus $48 \mathrm{~h}$ after hospitalization. Primary outcome was discharged to home/home health and secondary outcome was longitudinal CRP levels post CCP infusion.

Sarasota Memorial Hospital Institutional Review Board authorized consenting COVID-19 inpatients to participate in the national CCP protocol. Written informed consent was obtained from every CCP recipient or their legal guardian. Internal medicine resident physicians identified, contacted, and facilitated logistical pathways for CCP donation in collaboration with the community blood bank.

Enrolled patients were at least 18 years old with laboratory confirmed SARS-CoV- 2 infection admitted for treatment of severe or life-threatening COVID-19. Severe disease was defined as the presence of at least one of the following characteristics: dyspnea, respiratory rate of 30 breaths per minute or more, oxygen saturation (SpO2) equal to or less than 93\%, partial pressure of arterial oxygen to fraction of inspired oxygen less than 300 or development of lung infiltrates with more than $50 \%$ involvement within $24-48$ hours (h). Life-threatening disease was defined as the development of at least one of the following: respiratory failure, septic shock or multiple organ dysfunction or failure.

\section{Data analysis}

Analyses contrasted patients who underwent CCP infusion within or more than $48 \mathrm{~h}$ after admission. Primary outcome was discharged to home/home health. Continuous data summarized as median (interquartile range [IQR]) were compared using Kruskal-Wallis test or two-way analysis of variance (ANOVA). Discrete data were compared with Pearson's chi-square test. Two-tailed $\mathrm{p}<0.05$ was significant.

\section{Results}

The study included 197 patients consecutively admitted between March 28, 2020 and August 5, 2020. Of those, 92 received CCP infusion within $48 \mathrm{~h}$ and 105 received CCP infusion after $48 \mathrm{~h}$. Median age in the group that received CCP within $48 \mathrm{~h}$ was 67 years and 63 years in the group who received CCP after $48 \mathrm{~h}$. Of the 197 patients, $57 \%$ were male. Intergroup comorbidities were distributed similarly among both groups for the exception of body mass index (BMI) (32.7 (27-40)) vs. $\left(29.4(26-37) \mathrm{kg} / \mathrm{m}^{2}, \mathrm{p}<0.0001\right)$ (Table 1). Distribution of COVID-19 directed pharmacologic treatment was also similar amongst both groups.

\begin{tabular}{|c|c|c|}
\hline \multirow{2}{*}{ Characteristics } & Convalescent plasma $>48$ hours & Convalescent plasma $<48$ hours \\
\hline & $(\mathrm{N}=105)$ & $(\mathrm{N}=92)$ \\
\hline Age, median (IQR), & 67 (59-78) & $63(50-71)$ \\
\hline \multicolumn{3}{|l|}{ Sex, No. (\%) } \\
\hline Male & $59(56)$ & $54(59)$ \\
\hline Female & $46(44)$ & $38(41)$ \\
\hline \multicolumn{3}{|l|}{ Race, No. (\%) } \\
\hline White & $66(63)$ & $57(62)$ \\
\hline Black & $15(14)$ & $9(10)$ \\
\hline Other & $24(23)$ & $26(28)$ \\
\hline \multicolumn{3}{|c|}{ Anthropometrics, median (IQR) } \\
\hline Height (cm) & $166(160-177)$ & $168(161-178)$ \\
\hline Weight (kg) & $87.4(70.6-104.0)$ & $97.3(78.0-112.2)$ \\
\hline BMI $\left(\mathrm{kg} / \mathrm{m}^{2}\right)$ & $29.4(26-37)$ & $32.7(27-40)$ \\
\hline \multicolumn{3}{|l|}{ Vital signs, median (IQR) } \\
\hline Body temperature $\left({ }^{\circ} \mathrm{F}\right)$ & $98.3(98.0-98.9)$ & $98.5(98.1-99.0)$ \\
\hline Inspired $\mathrm{O}_{2}(\%)$ & $70(51-90)$ & $80(58-100)$ \\
\hline $\mathrm{SpO}_{2}(\%)$ & 95 (93-97) & 95 (93-97) \\
\hline
\end{tabular}




\section{Cureus}

\begin{tabular}{|c|c|c|}
\hline Respiratory rate, /min & $19(18-21)$ & $20(19-21)$ \\
\hline Heart rate, /min & $77(66-86)$ & $80(70-93)$ \\
\hline Systolic blood pressure, $\mathrm{mmHg}$ & $129(117-143)$ & $129(117-140)$ \\
\hline \multicolumn{3}{|l|}{ Coexisting diseases, No. (\%) } \\
\hline Hypertension & $60(59)$ & $38(43)$ \\
\hline Diabetes & $43(41)$ & $28(30)$ \\
\hline Obesity & $43(41)$ & $45(51)$ \\
\hline Kidney disease & $19(19)$ & $18(20)$ \\
\hline Depression & $16(16)$ & $12(14)$ \\
\hline Neurological disease & $14(14)$ & $13(15)$ \\
\hline Congestive heart failure & 11(11) & $9(10)$ \\
\hline \multicolumn{3}{|l|}{ COVID-19 pharmacotherapy } \\
\hline Dexamethasone & $86(82)$ & $73(79)$ \\
\hline Remdesivir & $65(62)$ & $64(70)$ \\
\hline Azithromycin & $17(16)$ & $21(23)$ \\
\hline Tocilizumab & $13(12)$ & $12(13)$ \\
\hline Hydroxychloroquine & $7(7)$ & $4(4)$ \\
\hline \multicolumn{3}{|l|}{ Laboratory tests, median (IQR) } \\
\hline \multicolumn{3}{|l|}{ Inflammatory biomarkers } \\
\hline CRP, mg/dL & $9.3(4.3-15.6)$ & $11.90(7.03-15.90)$ \\
\hline Ferritin, ng/mL & $592(302-1165)$ & $657(259-1290)$ \\
\hline Lactate dehydrogenase, U/L & $326(274-428)$ & $366(301-469)$ \\
\hline Procalcitonin, ng/mL & $0.12(0.05-0.98)$ & $0.14(0.05-0.59)$ \\
\hline Triglycerides, mg/dL & 200(118-297) & $161(99-236)$ \\
\hline \multicolumn{3}{|l|}{ Liver and kidney function } \\
\hline \multicolumn{3}{|l|}{ Aminotransferase, U/L } \\
\hline Alanine & $38(22-60)$ & $39(23-68)$ \\
\hline Aspartate & $42(29-61)$ & $41(31-68)$ \\
\hline Creatinine, mg/dL & $1.1(0.8-1.5)$ & $1.1(0.9-1.4)$ \\
\hline \multicolumn{3}{|l|}{ Complete blood cells count } \\
\hline White blood cells, /mL & $6.80(5.20-10.10)$ & $7.05(5.35-9.08)$ \\
\hline Neutrophil, /mL & $5.18(3.70-7.95)$ & $5.49(3.90-7.20)$ \\
\hline Lymphocyte, /mL & $19(12-28)$ & $18(12-25)$ \\
\hline Hemoglobin, gm/dL & $13.2(11.3-14.7)$ & $12.1(14.2-15.4)$ \\
\hline \multicolumn{3}{|l|}{ Coagulation profiles } \\
\hline D-dimer, mg/mL & $1.00(0.72-2.04)$ & $1.02(0.66-2.01)$ \\
\hline Prothrombin time, s & $11.3(10.8-12.0)$ & $11.4(10.7-12.3)$ \\
\hline aPTT, s & $30.2(27.3-32.9)$ & $30.8(26.4-32.9)$ \\
\hline
\end{tabular}




\section{Cureus}

Creatine Kinase Total, U/L

Troponin I, ng/mL

Pro-BNP, pg/mL
$131(63-271)$

$0.02(0.02-0.13)$

$1071(219-3141)$
$132(70-381)$

$0.02(0.02-0.06)$

225 (58-3004)

\section{TABLE 1: Baseline characteristics of consecutive patients with COVID-19.}

IQR: interquartile range; N: number; BMI: body mass index; CRP: C-reactive protein; s: seconds; SpO2: oxygen saturation; aPTT: activated partial thromboplastin time.

Initial admission vital signs and laboratory test results were not different between groups ( $>$ >.05) including temperature (98.4 (98.0-99.0) $\left.{ }^{\circ} \mathrm{F}\right)$; SpO2 (95, (93-97) \%); C-reactive protein (CRP) $(2.6(0.3-2.7) \mathrm{mg} / \mathrm{dL})$; Ddimer (1.01 (0.69-2.03) mg/L); and ferritin (624 (281-1228) ng/mL) (Figure 1).

ICCP within $48 \mathrm{~h}$ of Admission

-CCP after 48h of Admission
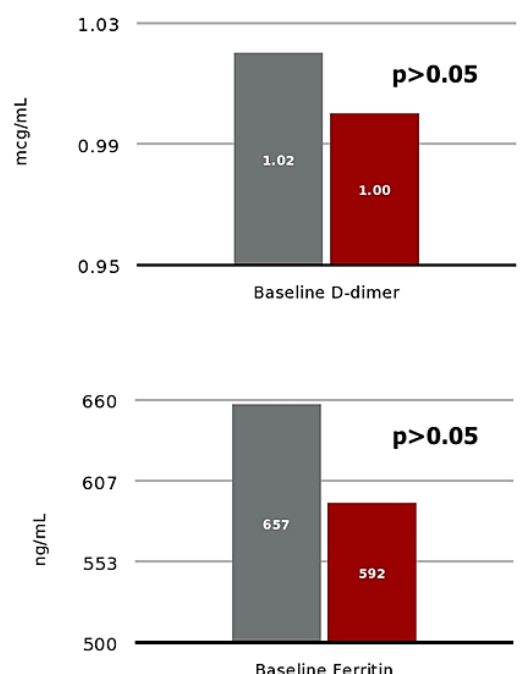

Baseline Ferritin
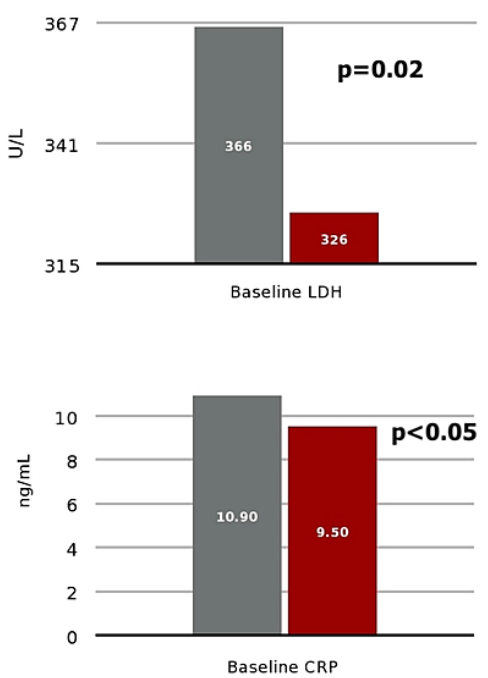

Baseline CRP

FIGURE 1: Baseline values for D-dimer, LDH, ferritin and CRP, respectively, for patients who received CCP within $48 \mathrm{~h}$ of admission vs. 48h after admission.

LDH: lactate dehydrogenase; CRP: C-reactive protein; CCP: COVID-19 convalescent plasma.

Time from admission to CCP infusion was 32.4 (22.0-41.2) vs. 81.7 (61.3-130.0) hours who respectively received CCP infusion within vs after 48h, p < 0.0001. Admission lactate dehydrogenase (LDH) was (366 (301469) U/L) vs. (326 (274-428) U/L, p = 0.02) (Figure 2). 


\section{Cureus}

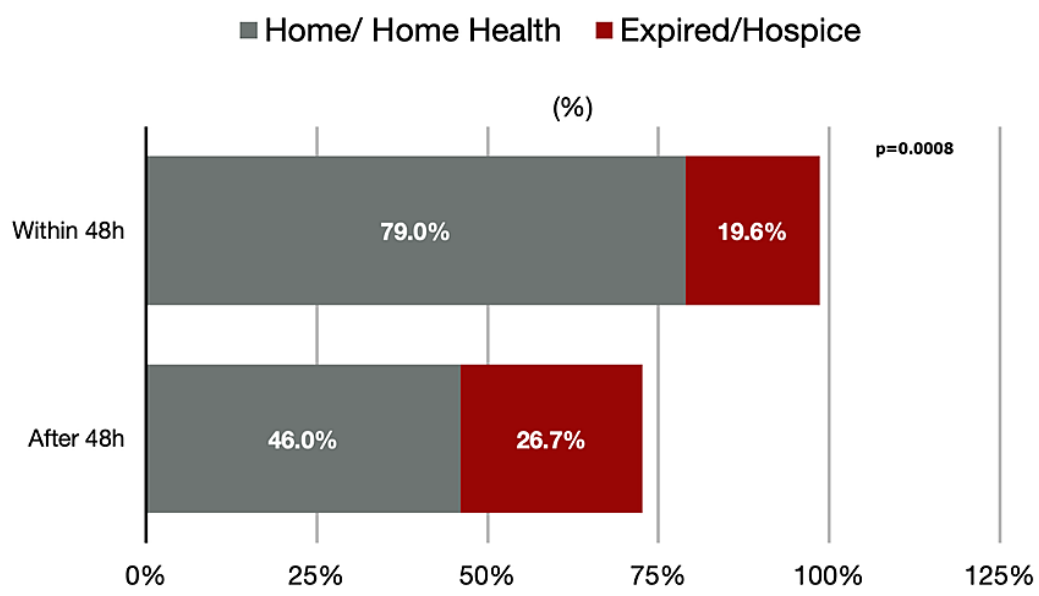

FIGURE 2: Percentage of patients discharged home/home health vs. expired/hospice.

Patients intubated included 25/92 (27\%) vs. 29/105 (28\%), $\mathrm{p}=0.34$. Days of mechanical ventilation were 8.0 (5.0-11.8) vs. 11.3 (4.5-17.9), $\mathrm{p}=0.16$. Hospital length of stay was $8.5(4.9-15.2)$ vs. $13.0(6.5-18.9)$ days, $\mathrm{p}=$ 0.03 (Table 2). Longitudinal CRP levels within $48 \mathrm{~h}$ pre-, $24 \mathrm{~h}$ post- and $25-48$ post-CCP infusion, respectively, were $(10.9(7.1-16.7)$ vs. $9.5(3.9-15.9))(p=0.12), 7.5(4.9-15.1)$ vs. $6.9(3.3-12.6)(p=0.20)$, and $4.4(2.7-9.0)$ vs. $6.1(4.0-12.0),(p=0.02)$ (Figure 3).

\begin{tabular}{|c|c|c|}
\hline \multirow{2}{*}{ Clinical outcomes } & Convalescent plasma $>48 \mathrm{~h}$ & Convalescent plasma $<48 \mathrm{~h}$ \\
\hline & $(N=105)$ & $(\mathrm{N}=92)$ \\
\hline ICU admission, No. (\%) & $40(38)$ & $34(37)$ \\
\hline ICU days, median (IQR) & $9.1(3.0-16.6)$ & $7.8(2.9-12.1)$ \\
\hline Intubation, №. (\%) & $30(29)$ & $23(25)$ \\
\hline Ventilation days, median (IQR) & $11.3(4.5-17.9)$ & $8.0(5.0-11.8)$ \\
\hline Survivor not Hospice LOS & $11.0(5.9-18.7)$ & $7.0(4.7-12.9)$ \\
\hline Overall LOS, median (IQR) & $13.0(6.5-18.9)$ & $8.5(4.9-15.2)$ \\
\hline Discharge to home, No. (\%) & $51(49)$ & $67(73)$ \\
\hline Expired or Hospice DC, No. (\%) & $28(26.7)$ & $18(19.6)$ \\
\hline
\end{tabular}

\section{TABLE 2: Outcomes.}

ICU: intensive care unit; LOS: length of stay; $\mathrm{N}=$ number; IQR: interquartile range; DC: discharge. 


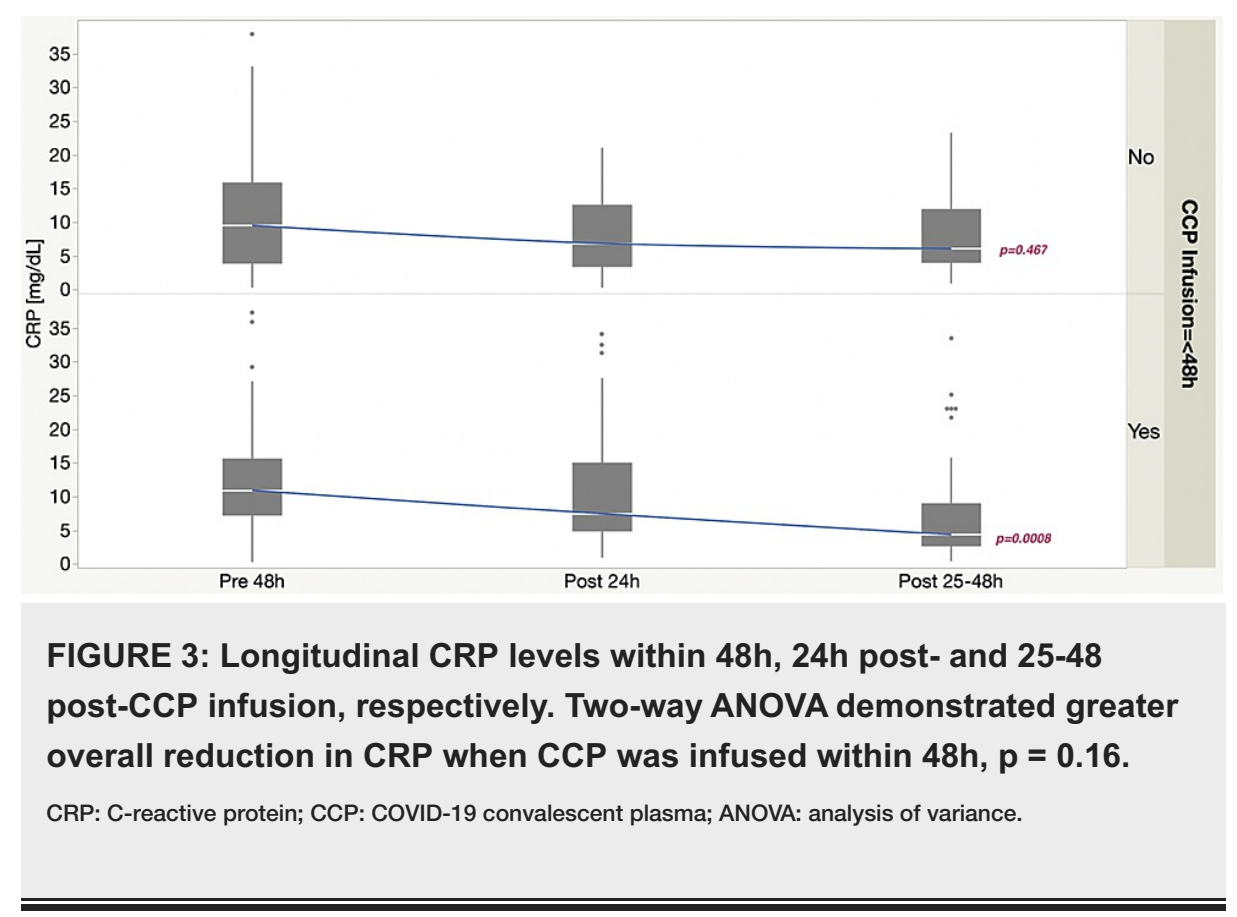

Primary outcome was discharged to home/home health which was 73/92 (79\%) vs. 49/105 (46\%), p = 0.005.

\section{Discussion}

Between March 28 and August 5, 2020, Sarasota Memorial Hospital participated in the Mayo Clinic-led national FDA expanded access program providing access to convalescent plasma protocol. Throughout this period, data from 197 participants were analyzed and contrasted among those who received CCP infusion within or more than $48 \mathrm{~h}$ after admission with a confirmed COVID-19 infection. We observed a post-CCP reduction in C-reactive protein, lower hospital length of stay and increase in discharge directly from hospital to home/home health in patients who received CCP within $48 \mathrm{~h}$ of admission rather than later in hospitalization. Moreover, earlier CCP treatment resulted in 7\% fewer patients who died or were discharged to hospice.

In December 2019, a new member of Coronaviridae family, SARS-CoV-2 was detected in Wuhan, China primarily manifesting as a respiratory illness [3]. Since then, multiple studies have been conducted exploring varied treatments for this lethal disease which has led to nearly 114 million of positive COVID-19 cases and 500,000 deaths in the United States. Presented with state of emergency accompanied by no available treatment and scarce medical resources; multiple centers around the world instituted CCP in a relatively timely manner previously shown effective during the SARS-CoV and Spanish Flu pandemic [3].

Initial evidence demonstrated CCP to be most beneficial when administered soon after SARS-CoV-2 infection. A randomized, double-blinded, placebo-controlled trial evaluated disease progression in 80 patients who received convalescent plasma within $72 \mathrm{~h}$ after onset of mild COVID-19 symptoms vs. placebo [9]. Disease progression was advancement to severe respiratory disease defined as respiratory rate more than 30 breaths per min and/or arterialized blood oxygen saturation less than $93 \%$ breathing ambient air. Of the 80 patients, $31 \%$ of the patients who received placebo vs. $16 \%$ who received CCP progressed to severe respiratory disease [9]. Life-threatening respiratory disease was observed in only $5 \%$ of CCP patients vs. $12 \%$ in the placebo group. This study concluded that the administration of CCP to infected patients within $72 \mathrm{~h}$ after the onset of symptoms reduce the risk of progression to severe respiratory disease by $48 \%$ [9]. CCP has demonstrated benefit in the clearance of SARS-CoV-2 in individuals who are symptomatic with COVID-19, including immunocompromised patients [8,10,11,12]. A cohort study of 966 patients with hematologic cancer and COVID-19 showed that after CP treatment, there was a significantly improved 30-day mortality [13].

CCP transfusion is associated with a reduction in inflammatory markers, such as CRP $[8,10,14]$. In a case series, 20 patients were treated with CCP compare to 20 controls matched with severe or life-threatening COVID-19 infection [15]. Results showed a marked reduction in CRP levels seven days after CCP infusion compared to control group. Discharges were similar amongst both groups, but mortality was higher in the control group. No patient died if they received CCP prior to seven days of hospitalization [15]. Another study reported lack of benefit when CCP was administered later (median of 21.5 days after diagnosis) for SARSCoV-2, which supports that earlier treatment may be of critical importance [16]. Our longitudinal CRP observations corroborate and extend evidence favoring least "door to treatment" time. 
Our data should be interpreted with some caveats. We conducted a monocenter pragmatic investigation. CCP was administered before FDA required titer labeling. Therefore, we couldn't establish if antibody titers in CCP transfused across patient groups were equivalently distributed. In addition, SARS-CoV-2 serologic testing was unreliable restricting the assessment of whether a patient exhibited an impaired humoral response.

\section{Conclusions}

Our study was conducted while no anti-viral treatment was approved by the FDA for patients hospitalized with COVID-19. We evince CCP treatment within $48 \mathrm{~h}$ of admission was associated a reduction in hyperinflammation and hospital length of stay in patients more obese with higher LDH levels with greater benefit for discharge to home/home health benefit and reduction in composite outcome of hospital mortality or discharge to hospice. Convalescent plasma has shown to be an effective treatment when given soon after SARS-CoV-2 infection. Unfortunately, in hospitalized patients with COVID-19, infusion of CCP late in the course of illness provides no observed benefit, as reported with other anti-viral agents.

\section{Additional Information \\ Disclosures}

Human subjects: Consent was obtained or waived by all participants in this study. Mark J. Magenheim, MD MPH, Medical Director IRB and Ashley Butler, CIP, Coordinator of the Institutional Review Board issued approval 21-COVID-30. Thank you for your submission of New Project materials for this research study. This research study qualifies for Expedited Review, as it involves no greater than minimal risk to subjects, and involves a retrospective review of medical records. The primary purpose of this study was to assess the relative impact of time from hospitalization to infusion of convalescent COVID-19 antibody-positive plasma (CCP) versus putative blood biomarkers of inflammation and organ injury evoked by patients hospitalized with a CoV-2 infection. Sarasota Memorial Hospital Institutional Review Board has APPROVED your submission. This approval is based on an appropriate risk/benefit ratio and a study design wherein the risks have been minimized. All research must be conducted in accordance with this approved submission. Animal subjects: All authors have confirmed that this study did not involve animal subjects or tissue. Conflicts of interest: In compliance with the ICMJE uniform disclosure form, all authors declare the following: Payment/services info: All authors have declared that no financial support was received from any organization for the submitted work. Financial relationships: All authors have declared that they have no financial relationships at present or within the previous three years with any organizations that might have an interest in the submitted work. Other relationships: All authors have declared that there are no other relationships or activities that could appear to have influenced the submitted work.

\section{Acknowledgements}

The authors would like to thank Sarasota Memorial Hospital and the Internal Medicine Residency for all their contributions in making this manuscript possible.

\section{References}

1. Rajendran K, Krishnasamy N, Rangarajan J, Rathinam J, Natarajan M, Ramachandran A: Convalescent plasma transfusion for the treatment of COVID-19: systematic review. J Med Virol. 2020, 92:1475-83. 10.1002/jmv.25961

2. Luke TC, Kilbane EM, Jackson JL, Hoffman SL: Meta-analysis: convalescent blood products for Spanish influenza pneumonia: a future H5N1 treatment?. Ann Intern Med. 2006, 145:599-609. 10.7326/0003-4819145-8-200610170-00139

3. Rojas M, Rodríguez Y, Monsalve DM, et al.: Convalescent plasma in Covid-19: possible mechanisms of action. Autoimmun Rev. 2020, 19:102554. 10.1016/j.autrev.2020.102554

4. Hung IF, To KK, Lee CK, et al.: Convalescent plasma treatment reduced mortality in patients with severe pandemic influenza A (H1N1) 2009 virus infection. Clin Infect Dis. 2011, 52:447-56. 10.1093/cid/ciq106

5. Expanded Access to Convalescent Plasma for the Treatment of Patients with COVID-19 . (2020). Accessed: 30 May 2021: https://www.uscovidplasma.org/-/media/kcms/gbs/patientconsumer/documents/2020/05/27/21/23/covid-19-plasma-eap.pdf.

6. Imai M, Iwatsuki-Horimoto K, Hatta M, et al.: Syrian hamsters as a small animal model for SARS-CoV-2 infection and countermeasure development. Proc Natl Acad Sci U S A. 2020, 117:16587-95. 10.1073/pnas.2009799117

7. Sun J, Zhuang Z, Zheng J, et al.: Generation of a broadly useful model for COVID-19 pathogenesis, vaccination and treatment. Cell. 2020, 182:734-743.e5. 10.1016/j.cell.2020.06.010

8. Klassen SA, Senefeld JW, Johnson PW, et al.: The effect of convalescent plasma therapy on mortality among patients with COVID-19: systematic review and meta-analysis. Mayo Clin Proc. 2021, 96:1262-75. 10.1016/j.mayocp.2021.02.008

9. Libster R, Pérez Marc G, Wappner D, et al.: Early high-titer plasma therapy to prevent severe Covid-19 in older adults. N Engl J Med. 2021, 384:610-8. 10.1056/NEJMoa2033700

10. Tremblay D, Seah C, Schneider T, et al.: Convalescent plasma for the treatment of severe COVID-19 infection in cancer patients. Cancer Med. 2020, 9:8571-8. 10.1002/cam4.3457

11. Robilotti EV, Babady NE, Mead PA, et al.: Determinants of COVID-19 disease severity in patients with cancer. Nat Med. 2020, 26:1218-23. 10.1038/s41591-020-0979-0 


\section{Cureus}

12. Senefeld JW, Klassen SA, Ford SK, et al.: Therapeutic use of convalescent plasma in COVID-19 patients with immunodeficiency. [Published online November 10]. BMJ. 2020, 10.1101/2020.11.08.20224790

13. Thompson MA, Henderson JP, Shah PK, et al.: Association of convalescent plasma therapy with survival in patients with hematologic cancers and COVID-19. JAMA Oncol. 2021, 10.1001/jamaoncol.2021.1799

14. Bandopadhyay P, Rozario R, Lahiri A, et al.: Nature and dimensions of the systemic hyper-inflammation and its attenuation by convalescent plasma in severe COVID-19. J Infect Dis. 2021, 10.1093/infdis/jiab010

15. Hegerova L, Gooley TA, Sweerus KA, et al.: Use of convalescent plasma in hospitalized patients with COVID-19: case series. Blood. 2020, 136:759-62. 10.1182/blood.2020006964

16. Wooding DJ, Bach H: Treatment of COVID-19 with convalescent plasma: lessons from past coronavirus outbreaks. Clin Microbiol Infect. 2020, 26:1436-46. 10.1016/j.cmi.2020.08.005 